\title{
Initial Rotor Position Estimation and Sensorless Direct Torque Control of Surface Mounted Permanent Magnet Synchronous Motors Considering Saturation Saliency
}

\author{
Ying Yan, Jianguo Zhu, and Youguang Guo \\ Faculty of Engineering, University of Technology, Sydney, P.O. Box 123, NSW 2007, Australia \\ E-mail: yingyan@eng.uts.edu.au, joe@eng.uts.edu.au, youguang@eng.uts.edu.au
}

\begin{abstract}
For a p ractical direct torque controlled (DTC) permanent magnet synchronous motor (PMSM) drive system, the information of the initial rotor position, which is usually obtained by a mechanical position sensor, is essential for starting under the full load. In order to avoid the disadvantages of using mechanical position sensors, great efforts have been made on the development of sensorless control schemes. This paper presents an initial rotor position estimation strategy for a DTC PMSM drive based on a nonlinear model of PMSM incorporating both structural and saturation saliencies. In the new scheme, specially designed high voltage pulses are applied to amplify the saturation saliencies. The peak currents corresponding to the voltage pulses are used, in combination with the inductance patterns, to determine the $d$-axis position and the polarity of the rotor. The presented initial rotor position identification strategy has been implemented in a sensorless DTC drive for a surface mounted PMSM. Experiments are conducted to confirm the effectiveness of the method and the performance of the drive system.
\end{abstract}

Keywords: Permanent magnet synchronous motor (PMSM); surface mounted PMSM; direct torque control scheme; numerical simulation; saturation saliency; inductance; initial rotor position estimation. 


\section{Introduction}

For a permanent magnet synchronous motor (PMSM) drive, the rotor position information is essential for the inverter commutation to control the frequency and position of the stator current vectors. The rotor position information can be obtained by using mechanical position sensors or calculation with various sensorless schemes. With the wide application of the PMSM in different environments, sensorless schemes have become desirable for eliminating the handicaps of mechanical sensors, such as high system cost, limited operating condition, high maintaining requirement, and low system reliability. Among the modern control strategies adopted to drive the PMSM, the direct torque control (DTC) scheme is preferred because sensorless control scheme could be realised if the initial rotor position of the motor is known. Therefore, the accurate identification of the initial rotor position is crucial for a DTC PMSM drive.

The sensorless techniques can be roughly classified into two types: motional electromotive force (emf) and inductance variation [1]. As there is no motional emf induced at standstill, the method based on the motional emf cannot be applied to detect the initial rotor position. For the inductance variation method, the structural and magnetic saturation saliencies are employed, which occur in all types of PMSMs. Normally the current changing rate determined by the inductances as a function of rotor positions and stator currents can be directly and/or indirectly measured [2]. With the method, the initial rotor position can be accurately identified for the motors with large structural saliency, e.g. the interior PMSM. However, for the surface mounted PMSM, which does not have significant structural saliency, it has been a difficulty to detect the rotor position at zero or low speeds under load conditions. Numerous efforts have 
been made in this field by injecting signals to amplify the saturation saliency [3-8].

According to the types of the applied signals, the inductance variation detection technique can be broadly categorised as injecting either a high frequency signal [3-4] or a special pulse width modulation (PWM) signal [5-8]. The special PWM signal includes a series of voltage pulses [7-8]. By analysing the inductance variation due to saturation saliency in a surface mounted PMSM, the amplitude variation of the three phase peak currents caused by several voltage pulses was utilised to estimate the $d$-axis of the rotor and rotor polarity [7-8]. Compared with the high frequency signal injection method, the method using the voltage pulses offers a less expensive, less complex, and less maintenance solution.

In this paper, an initial rotor position estimation scheme using voltage pulses is presented. In order to numerically simulate the performance of new initial rotor position estimation, a nonlinear model of PMSMs which incorporates both structural and saturation saliencies has been investigated [9-12]. In the model, the self and mutual incremental inductances of the phase windings are expressed by Fourier series as functions of the rotor position and stator currents. With the model, the initial rotor position estimation method is numerically simulated. In this way, the effectiveness of the scheme could be analysed and evaluated before being implemented in the practical system. Experiments are also carried out. The validity of the proposed method is examined and confirmed through extensive experiments. Finally, the initial rotor position identification scheme is applied in a DTC scheme for implementation of a sensorless DTC drive system. The performance of the DTC scheme with the estimated initial rotor position is simulated, and the experiments have been conducted to verify the performance of the DTC PMSM drive system. 


\section{Nonlinear Model of PMSM with Saliencies}

\subsection{Flux linkage and circuit equations}

In a PMSM, the operating point of the magnetic core is mainly determined by the rotor magnetic field, which is the dominant component. Meanwhile, the magnetic field produced by the stator current also contributes to the total magnetic field. Therefore, as an example, there are two components in the total flux linkage of phase $a, \psi_{a}$. As described in (1), $\psi_{a s}$ and $\psi_{a f}$ are the flux linkage components produced by the magnetisation component of the stator currents, $i_{m s}$, and rotor magnets, respectively, and $\theta$ is the rotor position. The flux linkage $\psi_{a s}$ can be further separated into the three components, $\psi_{a a}, \psi_{a b}$ and $\psi_{a c}$, which depend on the three phase currents, $i_{a}, i_{b}, i_{c}$, and the equivalent current of the magnetic field due to the rotor permanent magnet, $i_{f}$. Although the $\psi-i$ characteristic of the magnetic core is nonlinear, the magnetic circuit can be considered as piecewisely linearised around the operating point corresponding to a given rotor position [9-12].

$$
\psi_{a}=\psi_{a s}+\psi_{a f}\left(i_{f}, \theta\right)=\psi_{a a}+\psi_{a b}+\psi_{a c}+\psi_{a f}\left(i_{f}, \theta\right)
$$

Under the assumption of the piecewisely linearization around the operating point, the self and mutual incremental inductances of phase $a$ winding are defined as the proportionality coefficients $L_{a a}, L_{a b}$ and $L_{a c}$ determined by the gradient of the magnetisation curve at the operating point. The flux linkages of phases $b$ and $c$ can be studied in the similar way. The voltage equations of the three phase windings can be described as,

$$
\left[\begin{array}{c}
v_{a} \\
v_{b} \\
v_{c}
\end{array}\right]=\left[\begin{array}{ccc}
R_{a} & 0 & 0 \\
0 & R_{b} & 0 \\
0 & 0 & R_{c}
\end{array}\right]\left[\begin{array}{c}
i_{a} \\
i_{b} \\
i_{c}
\end{array}\right]+\left[\begin{array}{ccc}
L_{a a} & L_{a b} & L_{a c} \\
L_{b a} & L_{b b} & L_{b c} \\
L_{c a} & L_{c b} & L_{c c}
\end{array}\right] \frac{d}{d t}\left[\begin{array}{c}
i_{a} \\
i_{b} \\
i_{c}
\end{array}\right]+\left[\begin{array}{c}
e_{a f}+e_{a \theta} \\
e_{b f}+e_{b \theta} \\
e_{c f}+e_{c \theta}
\end{array}\right]
$$


where $v_{a}, v_{b}$ and $v_{c}$ are the terminal voltages, $R_{a} R_{b}$ and $R_{c}$ are the winding resistances, $i_{a}, i_{b}$ and $i_{c}$ are the currents of phases $a, b$ and $c$, respectively, $e_{a f}=\omega d \psi_{a f} d \theta, e_{b f}=\omega d \psi_{b f} / d \theta$, and $e_{c f}=\omega d \psi_{c f} d \theta$ are the emfs induced by the rotor magnets, $\omega=d \theta / d t$ is the angular speed of the rotor, and $e_{a \theta}=\omega\left(\partial \psi_{a a} / \partial \theta+\partial \psi_{a b} / \partial \theta+\partial \psi_{a d} \partial \theta\right), e_{b \theta}=\omega\left(\partial \psi_{b a} / \partial \theta+\partial \psi_{b b} / \partial \theta+\partial \psi_{b c} / \partial \theta\right)$, and $e_{c \theta}=$ $\omega\left(\partial \psi_{c a} / \partial \theta+\partial \psi_{c b} / \partial \theta+\partial \psi_{c c} \partial \theta\right)$ are the three phase emfs induced by the variation of flux linkage due to the saliencies.

\subsection{Electromagnetic torque}

The electromagnetic torque of the PMSM can be obtained by taking the partial derivative of the system co-energy, $W_{f}^{\prime}$, with respect to the rotor position angle $\theta_{r}$, i.e. $T=\partial W_{f}^{\prime} / \partial \theta_{r}$, and it can be derived as [9-12],

$T_{e m}=i_{a} \frac{\partial \psi_{a f}}{\partial \theta_{r}}+i_{b} \frac{\partial \psi_{b f}}{\partial \theta_{r}}+i_{c} \frac{\partial \psi_{c f}}{\partial \theta_{r}}+\frac{i_{a}^{2}}{2} \frac{\partial L_{a a}}{\partial \theta_{r}}+\frac{i_{b}^{2}}{2} \frac{\partial L_{b b}}{\partial \theta_{r}}+\frac{i_{c}^{2}}{2} \frac{\partial L_{c c}}{\partial \theta_{r}}+i_{a} i_{b} \frac{\partial L_{a b}}{\partial \theta_{r}}+i_{a} i_{c} \frac{\partial L_{a c}}{\partial \theta_{r}}+i_{b} i_{c} \frac{\partial L_{b c}}{\partial \theta_{r}}$

There are two components of the electromagnetic torque. One is produced by the stator currents and rotor magnets, and the other is caused by the saliencies.

\section{Nonlinear Incremental Inductances}

\subsection{Experimental measurement of phase inductances}

In order to measure the self and mutual inductances, which could be incorporated in the presented model, various rotor and stator fluxes for emulating the operating conditions should be produced during the experiment. A special method is designed to measure the self and 
mutual inductances. By this method, not only the structural saliency but also the saturation saliency can be mathematically reflected in the measured inductances.

The experiment is carried out on a 6-pole off the shelf surface mounted PMSM, and Table 1 lists the nameplate data of the testing PMSM motor. Different DC offsets, which are used to emulate the saturation saliency of the magnetic core due to the three phase currents, are injected to one of the stator windings. Meanwhile, a small AC current is applied to one phase for measuring the inductances, while the other two phases are open-circuited. The power, voltage and current of the phase with the $\mathrm{AC}$ current are measured and the voltages of the other two phases are also recorded by a power analyser. The self and mutual inductances of phases $a, b$ and $c$ at different rotor positions and stator currents can then be calculated with the experimental results via circuit analysis [9-12].

Table I: Nameplate Data of a PMSM

\begin{tabular}{cc}
\hline \hline Parameters & Quantities \\
\hline \hline Model & IFT 6071-6AC21-2-Z \\
Number of poles & 6 \\
Number of phases & 3 \\
Rated power & $1000 \mathrm{~W}$ \\
Rated voltage (line to line) & $128 \mathrm{~V}$ \\
Rated current & $6.5 \mathrm{~A}$ \\
Rated speed & $2000 \mathrm{rev} / \mathrm{min}$ \\
Rated torque & $4.5 \mathrm{Nm}$ \\
\hline \hline
\end{tabular}

\subsection{Identification of nonlinear inductance with arbitrary rotor and stator flux}

Since the flux produced by the rotor permanent magnets of a PMSM can be considered as a constant, the inductances can be expressed as $L\left(\theta_{r}, \theta_{s},\left|\Phi_{s}\right|\right)$, where $\theta_{s}$ and $\left|\Phi_{s}\right|$ are the position and amplitude of the stator flux, and $\theta_{r}$ is the position of the rotor flux. In order to 
simplify the inductance expression and unify the definitions of $\theta_{s}$ and $\theta_{r}$, the angle between the stator flux, $\Phi_{s}$, and the stator winding axis of phase $a$, denoted by $\alpha$, and the angle between the stator and rotor fluxes, denoted by $\delta$, are used in the inductance expressions instead of $\theta_{s}$ and $\theta_{r}$. With the new definitions, the inductances can be expressed as $L\left(\delta, \alpha, i_{m s}\right)$, where $i_{m s}=0 \sim I_{\text {rated }}$ corresponds to various $\left|\Phi_{s}\right|$, as shown in Fig. 1 . In the figure, $\Phi_{s 1}, \Phi_{s 2}$ and $\Phi_{s 3}$ are the stator flux produced by a DC offset injected in phase $a, b$ and $c$, respectively, and $\Phi_{r}$ is the rotor flux at a given rotor position. Based on the finite experimental results at several rotor and stator fluxes, the analytical expressions of the nonlinear self and mutual inductances with a reasonable number of parameters at arbitrary rotor and stator flux can be estimated to incorporate the structural and saturation saliencies in the new model.

As discussed in [9-13], the phase inductances can be considered as a periodic function of rotor angular position and varies with the stator current due to the saturation saliency. A Fourier series is utilised to express the nonlinear inductances as,

$$
L\left(\delta, \alpha, i_{m s}\right)=a_{0}\left(\alpha, i_{m s}\right)+\sum_{m=1}^{n}\left(a_{m}\left(\alpha, i_{m s}\right) \cos (m \delta)+b_{m}\left(\alpha, i_{m s}\right) \sin (m \delta)\right)
$$

where the coefficients and the number of terms of the Fourier series can be determined by the curve fitting of the experimental phase inductances with sufficient accuracy.

According to the experiments with DC offsets in the stator winding of each phase, the inductances at $\alpha=0^{\circ}, 120^{\circ}$ and $240^{\circ}$ with several $\delta$ and $i_{m s}$ have been acquired. With (4), the self and mutual inductances at $\alpha=0^{\circ}, 120^{\circ}$ and $240^{\circ}$ with arbitrary $\delta$ and $i_{m s}$ can be obtained. Then, the interpolation technique is employed to estimate the inductance at arbitrary $\alpha, \delta$ and $i_{m s}$. Fig. 2 shows the $L_{b b}$ at different $\alpha$ and $\delta$ in an angular cycle with $i_{m s}=5.5 \mathrm{~A}$. 


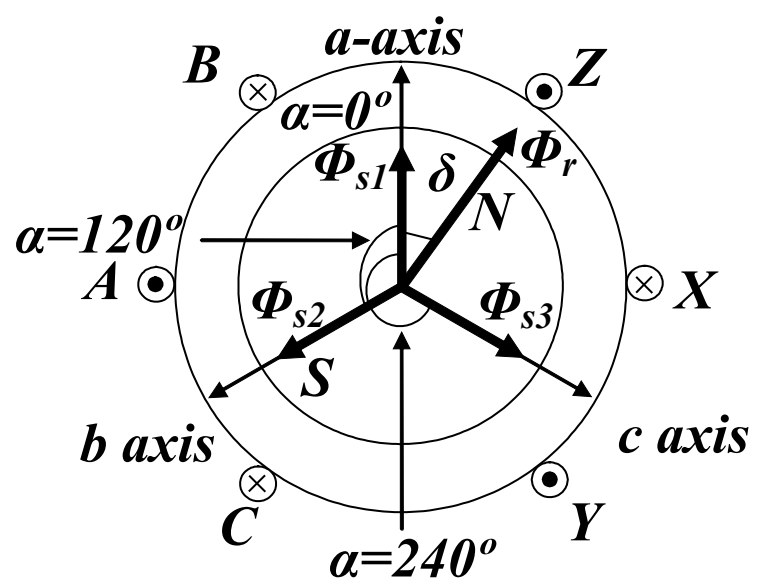

Fig. 1. Rotor and stator fluxes at a given rotor position with a DC offset in phases $a, b$ and $c$, respectively.

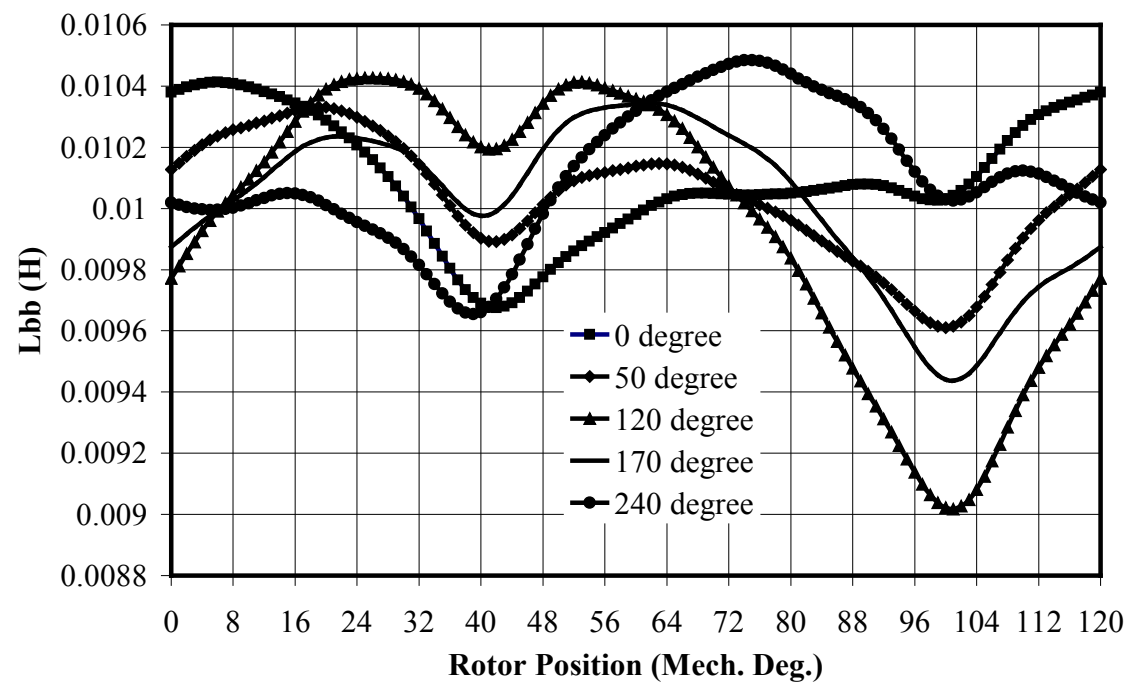

Fig. 2. Measured and calculated $L_{b b}$ at different $\alpha$ with a DC offset of 5.5A.

\section{Initial Rotor Position Estimation for a PMSM}

\subsection{Configuration of experimental setup and experimental principle}

In order to obtain the rotor position related saliency information, several voltage pulses with high amplitudes and short duty are injected to the stator windings. These pulse voltages are produced by PWM inverters in a drive system and no additional hardware is required. The 
gate signals of the inverters are controlled by a dSPACE DS1104 system. The three phase currents are detected by Hall-effect current probes and are recorded by a digital oscilloscope. The peak values of the three phase currents are used to estimate the initial rotor position. Meanwhile, the actual value of the rotor position is detected from an optical encoder mounted on the shaft of the motor for verifying the estimation and the resolution of the encoder is 2500 counts per revolution.

The fundamental principle for this scheme is as following. At an unknown initial rotor position, a positive pulse is applied to one of the three phase terminals for a certain duty, while the terminals of the other two phases are applied with negative pulses. With this positive pulse, the positive peak current value is acquired. After that, the positive pulse is followed by a negative pulse and the negative pulses by positive pulses to bring the phase currents down to zero. Meanwhile, at the same rotor position, a negative pulse is applied to the same phase for the same duty, while the terminals of the other two phases are supplied with positive pulses. In this way, the negative peak current value is measured. After that, one positive pulse and two negative pulses are used to bring the phase currents down to zero. For example, the gate signals of 010 are created to inject positive pulse to phase $b$ for a short period, and then immediately the gate signals of 101 are injected. After the stator currents become zero, the gate signals of 101 are created, and then immediately the gate signals of 010 are produced to drive the current in the phase winding to zero. 


\subsection{Factors determining peak currents}

For the measurement of the peak currents, the peak current value is very sensitive to the applied voltage pulse and resolutions of the current probes, oscilloscope and peripheral devices. In order to demonstrate these effects, a series of high voltage pulses with the same duty and amplitude are applied to the motor. The current responses are obtained, and the difference among these peak current values can be detected. Since the peak current values are directly used for initial rotor position identification, the detection of these values with sufficient precision is necessary. Therefore, the factors which might affect the measuring accuracy should be studied.

At first, the remanent magnetisation and magnetic hysteresis effects are analysed. Generally, there is some remanent magnetisation for magnetic core even without excitation currents. When a series of positive and negative voltage pulses are applied, the magnetic field is enforced or reduced in two directions. Even for the same type of voltage pulses at a given rotor position, the operating points of the magnetic field are different due to the remanent magnetisation and various path of the minor hysteresis loops, and hence the corresponding saturation saliencies are different. In order to avoid the peak current error due to remanent magnetisation and magnetic hysteresis effects, the number of voltage pulses should be increased to diminish the effects. Meanwhile, a series of additional positive and negative voltage pulses should be continuously applied before the peak current is measured.

Besides the magnetic characteristics, the duty and amplitude of the voltage pulse are also important. When the high voltage is applied in a short time, the amplitude of the voltage pulse should be kept as constant. To compensate the voltage drop of the PWM inverters during the 
rapid switching of the inverters, and to protect the inverters, some additional capacitors are used to stabilise the voltage pulses. At the same time, the duty of the voltage pulse should be carefully chosen, because the duty of the pulse is short and the length of the pulse will definitely affect the peak current values. In the testing system, a fixed sampling time has been determined as a multiple of the duty of the voltage pulses.

After the current responses have been obtained by the oscilloscope, the data will be transferred to a host computer via data communication channel. Since the number of the captured data is limited for the oscilloscope, only the current responses to the last few measuring voltage pulses are recorded by setting the trigger position to avoid this oscilloscope acquisition error, while the current responses with respect to the several additional positive and negative pulses applied to eliminate the effects of the remnant magnetisation and magnetic hysteresis are not recorded.

With the consideration of the factors mentioned above, a large amount of experiment is conducted for various types of voltage pulses, such as 12,24 a nd 36 positive and negative voltage pulses with adjustable duties. Finally, totally 36 voltage pulses with 18 positive and 18 negative ones are chosen, and only the current responses to the last four positive and negative pulses are captured, recorded and stored. As an example, the phase $a$ current responses to the applied voltage pulses are plotted in Fig. 3. From the peak current values determined by P1 to P4 or N1 to N4, it can be seen that the difference among the four peak current values is little. By injecting this specially designed voltage pulses to the motor, the effects due to the above factors could be neglected. At a fixed rotor position, these voltage pulses are injected to phase 
$a, b$ and $c$, respectively, and the corresponding currents are recorded and processed. The motor is mechanically rotated from one position to the next.

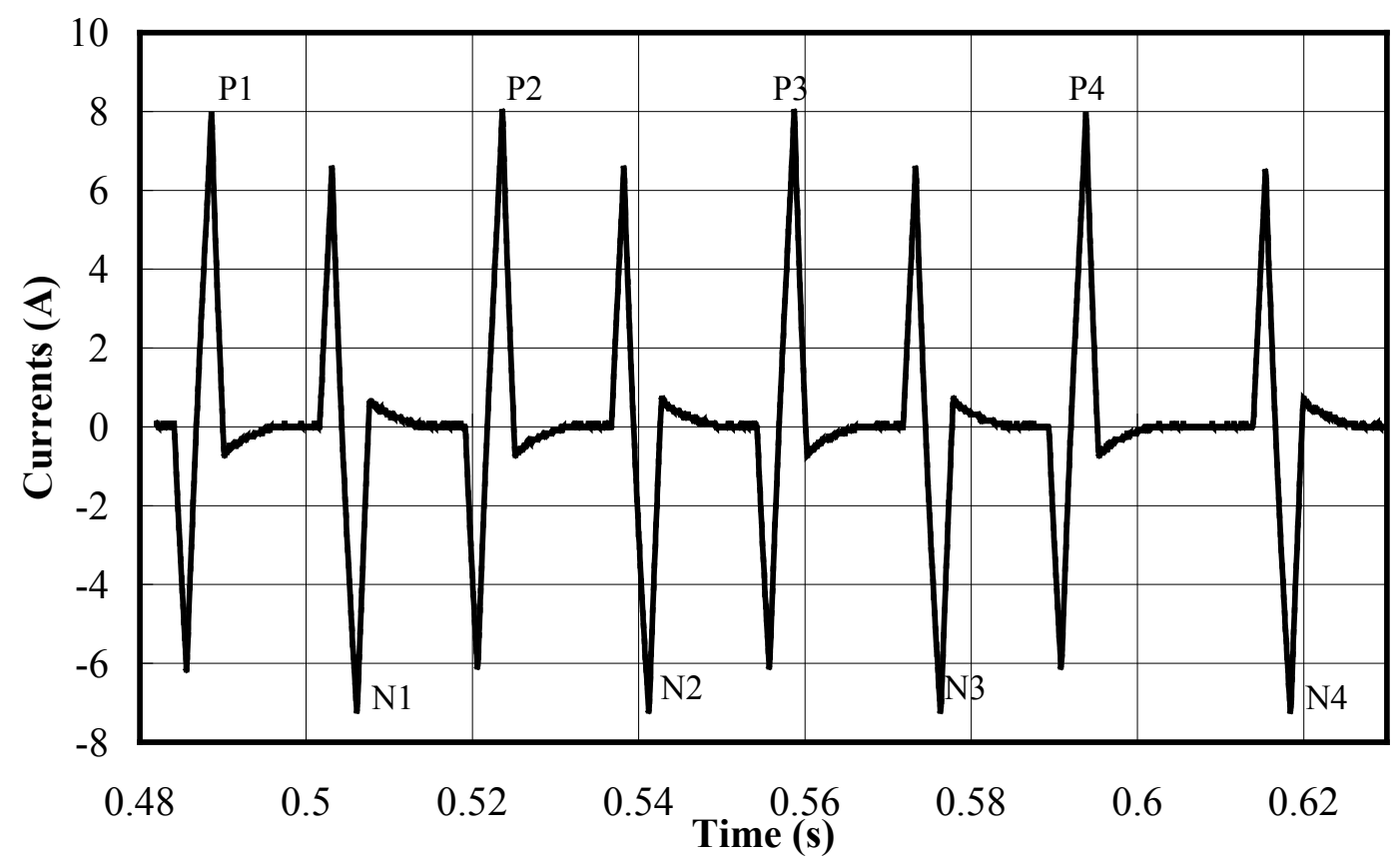

Fig. 3. Phase $a$ current responses to the last four positive and negative voltage pulses.

\subsection{Experimental peak current}

The voltage pulse injection experiment as discussed above is conducted for the testing surface mounted PMSM. At every rotor position, the average value of the last four peak currents is defined as the value of the positive (or negative) peak current. At a given rotor position, the six peak currents corresponding to the positive and negative voltage pulses injected to the three phase stator windings are plotted in Fig. 4. The positive and negative peak currents of phase $a, b$ and $c$ at different rotor positions are plotted in Fig. 5. 


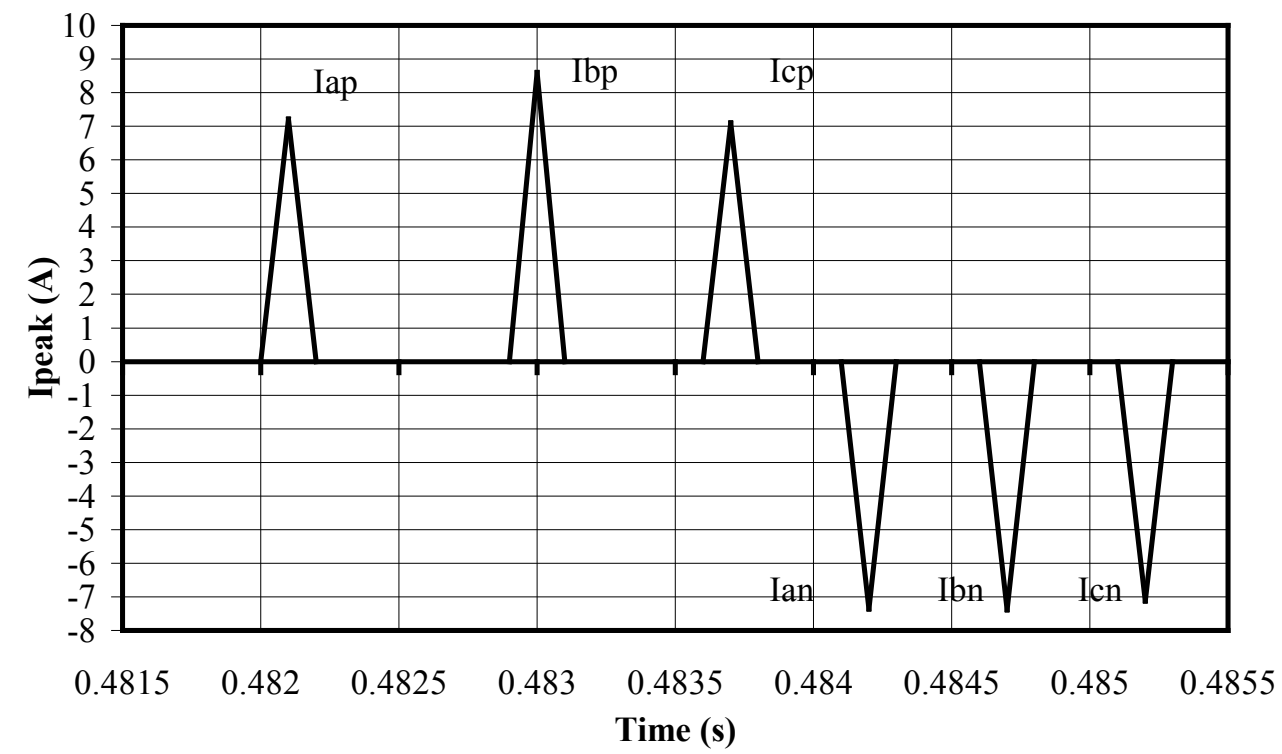

Fig. 4. Three phase positive and negative peak currents at a given rotor position.

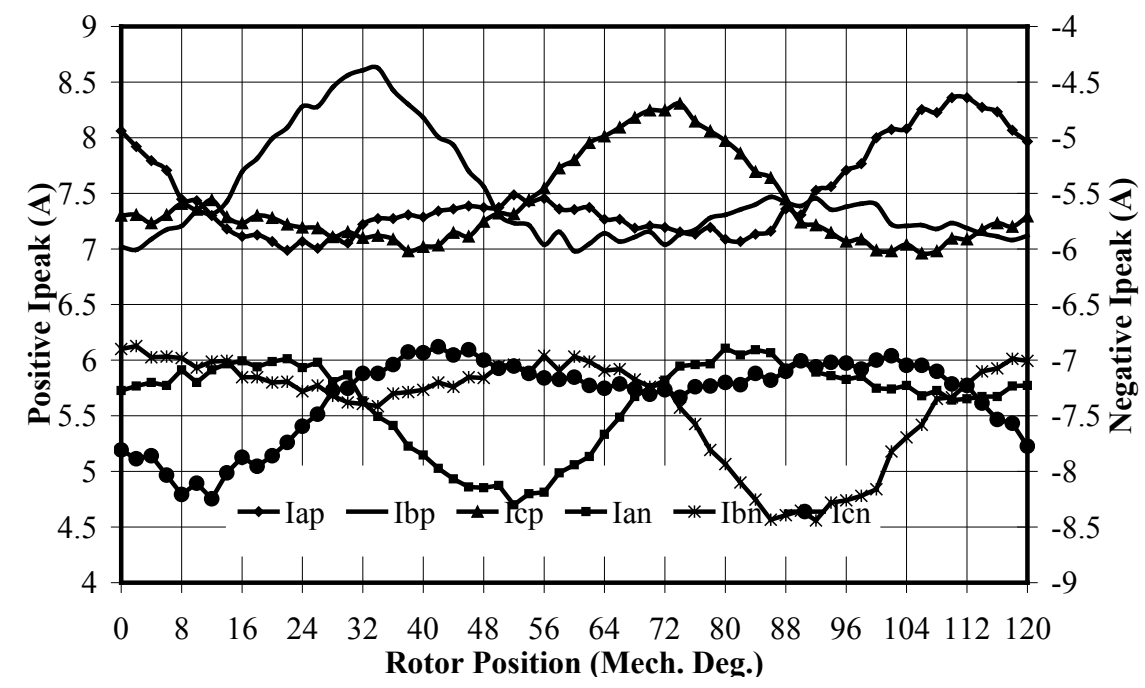

Fig. 5. Three phase peak currents versus rotor position.

\subsection{Initial rotor position estimation strategy}

From Fig. 5, the measured peak currents to positive and negative voltage pulses can be modelled by several periodic functions as $I_{a}=I_{0}+\Delta I_{01} \cos \theta+\Delta I_{01} \cos 2 \theta, \quad I_{b}=I_{0}+\Delta I_{01} \cos (\theta$ - 
$2 \pi / 3)+\Delta I_{01} \cos (2 \theta+2 \pi / 3), I_{c}=I_{0}+\Delta I_{01} \cos (\theta+2 \pi / 3)+\Delta I_{01} \cos (2 \theta-2 \pi / 3)$ [7-8]. At a given rotor position, the amplitude of the peak current variation, $\Delta i_{s p}$ and $\Delta i_{s n}$ plotted in Fig. $6 \mathrm{c}$ an be obtained as $\Delta i_{s p}=I_{s p}-I_{0 p}$, and $\Delta i_{s n}=I_{s n}-I_{O n}$, where the subscript $s$ represents phase $a, b$ and $c, I_{0 p}$ and $I_{0 n}$ are the average values of the three phase currents, and $I_{s p}$ and $I_{s n}$ the three phase peak current values. $\Delta i_{s p}$ and $\Delta i_{s n}$, caused by the saturation saliency at different rotor positions and stator currents, can be used to identify the rotor position. In order to identify the maximum values among $\Delta i_{s p}$ and $\Delta i_{s n}$ at a fixed rotor position, the absolute values of $\Delta i_{s p}$ and $\Delta i_{s n}$ are calculated [7-8].

With the amplitude of the peak current variation, the general procedure of initial rotor position estimation is given in Fig. 7. There are two steps for determining the rotor position at standstill. Firstly, the positive and negative voltage pulses in the proposed pattern are provided to each stator winding, and the peak current values of each phase have been selected. Based on the three phase positive and negative peak currents, the amplitude of the peak current variation, $\Delta i_{s p}$ and $\Delta i_{s n}$, have been obtained and rectified to their absolute values. Among the six values, the maximum one is chosen to identify the rotor position section, $\theta_{\text {section }}$, within an electrical cycle. Secondly, $\Delta i_{s p}$ and $\Delta i_{s n}$, which correspond to the other two phases except for the phase with the maximum peak current, are used to identify a subsection angle $\theta$. The final rotor position is determined by $\theta_{r}=\theta_{\text {section }}+\theta$. With the experimental three phase peak currents, the initial rotor positions are estimated and compared with the actual rotor positions as shown in Fig. 8. 


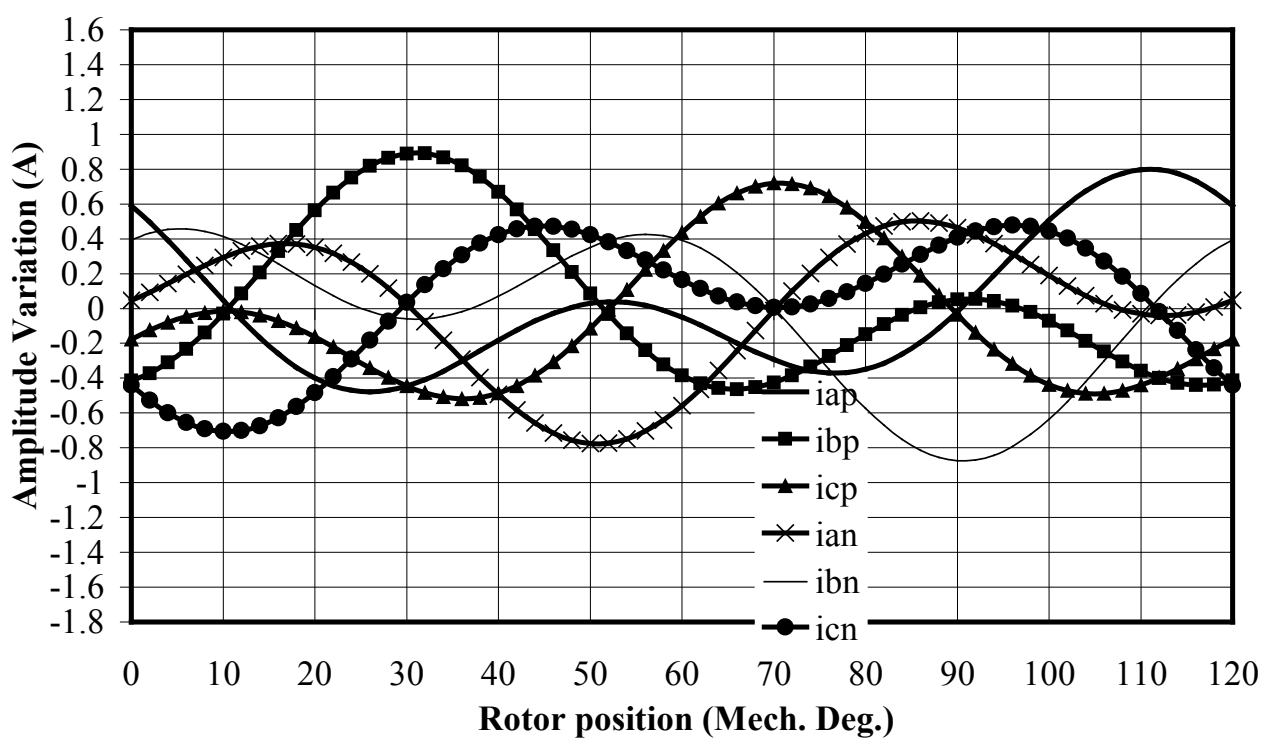

Fig. 6. The amplitude of the peak current variations versus rotor position. 


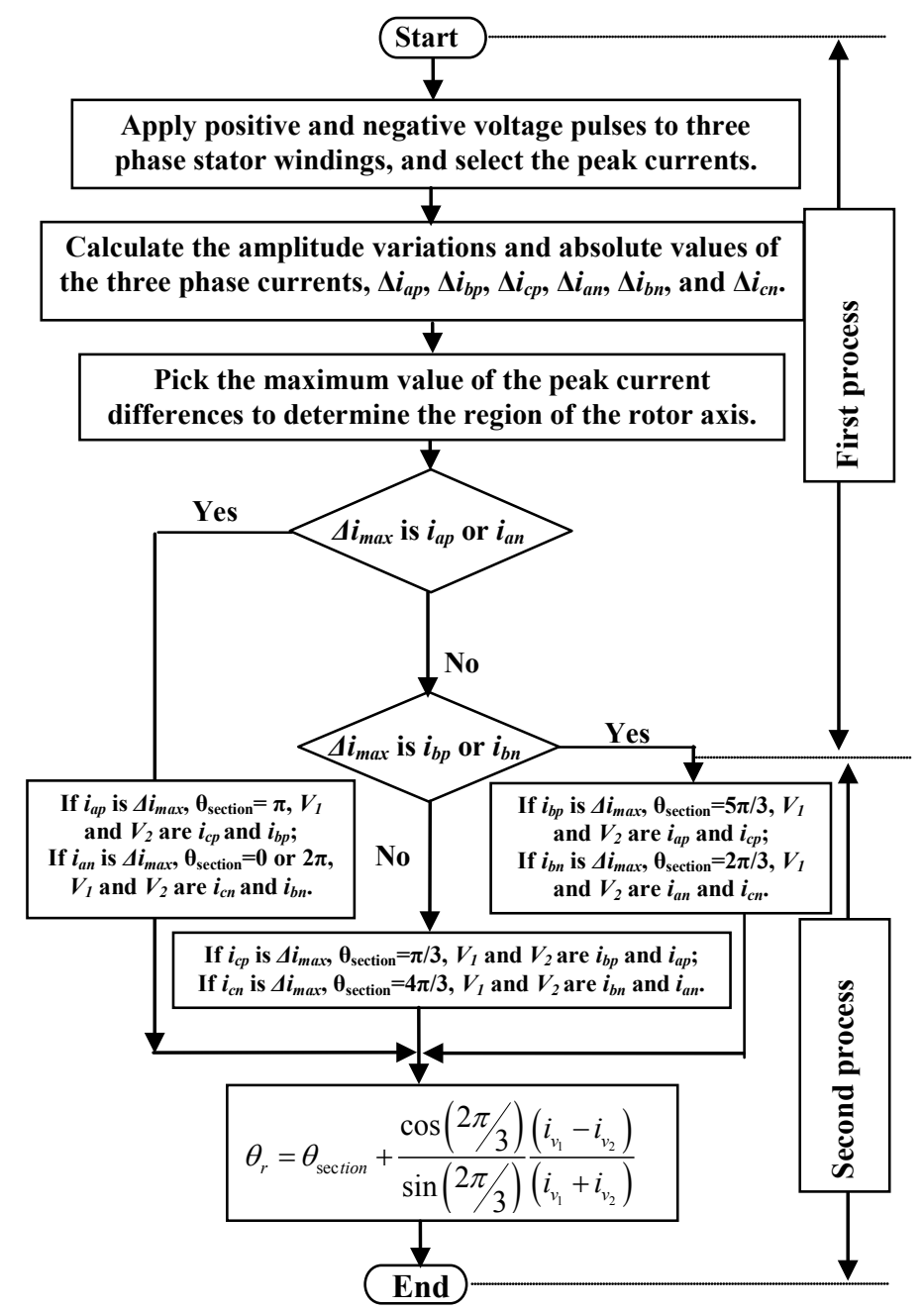

Fig. 7. Flow chart of the initial rotor position estimation.

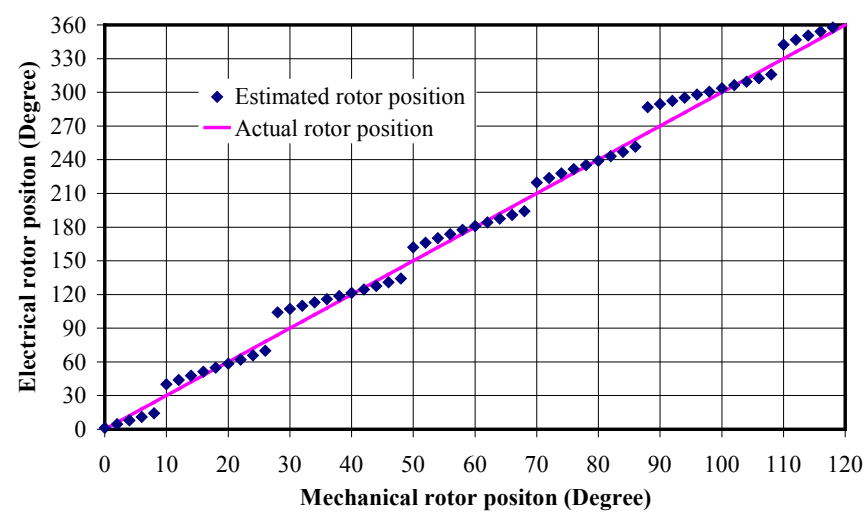

Fig. 8. Actual and estimated initial rotor position. 
To assess the accuracy of the scheme, the estimation error is defined as,

$$
E r r=\frac{\sqrt{\frac{1}{(N-1)} \sum_{i=1}^{N}\left(\theta_{i_{-} e s t}-\theta_{i_{-} a c t}\right)^{2}}}{\max \left\{\theta_{i_{-} a c t}\right\}}
$$

where $N$ is the number of actual rotor positions, $\theta_{i_{-} e s t}$ and $\theta_{i_{-} a c t}(i=1,2, \ldots, N)$ are the estimated and actual rotor position, respectively, and $\max \{\}$ is to choose the maximum value in the actual rotor positions. The error of the estimation results is calculated as $2.4 \%$ by (5). This algorithm can produce satisfactory results.

\section{Implementation of a Sensorless DTC Drive}

\subsection{Sensorless DTC drive system}

It has been mentioned that in a DTC PMSM drive system, it is possible to drive the motor without mechanical position sensors. In the conventional DTC, the amplitude of the stator flux is maintained as constant, and the torque is adjusted by tuning the load angle. In this scheme, only six voltage vectors are produced by the inverters. Normally, the DTC algorithm is implemented in the two phase stationary $\alpha-\beta$ reference frame. The $\alpha$ - and $\beta$-axis stator fluxes are calculated as [14],

$$
\psi_{m}\left(t_{n+1}\right)=\left(u_{m}\left(t_{n}\right)-R_{s} i_{m}\left(t_{n}\right)\right) T_{s}+\psi_{m}\left(t_{n}\right)
$$

where the subscript $m$ refers to $\alpha$ and $\beta, T_{s}=t_{n+1}-t_{n}$ is the sampling time in the digital control system, and $R_{S}$ is the phase resistance. Meanwhile, the electromagnetic torque is calculated by,

$$
T_{e m}\left(t_{n}\right)=\frac{3}{2} P\left(\psi_{\alpha} i_{\beta}-\psi_{\beta} i_{\alpha}\right)
$$


where $P$ is the number of pole pairs.

With the presented initial rotor position scheme, a sensorless DTC drive for the testing surface mounted PMSM is presented and the block diagram of the drive system is shown in Fig. 9.

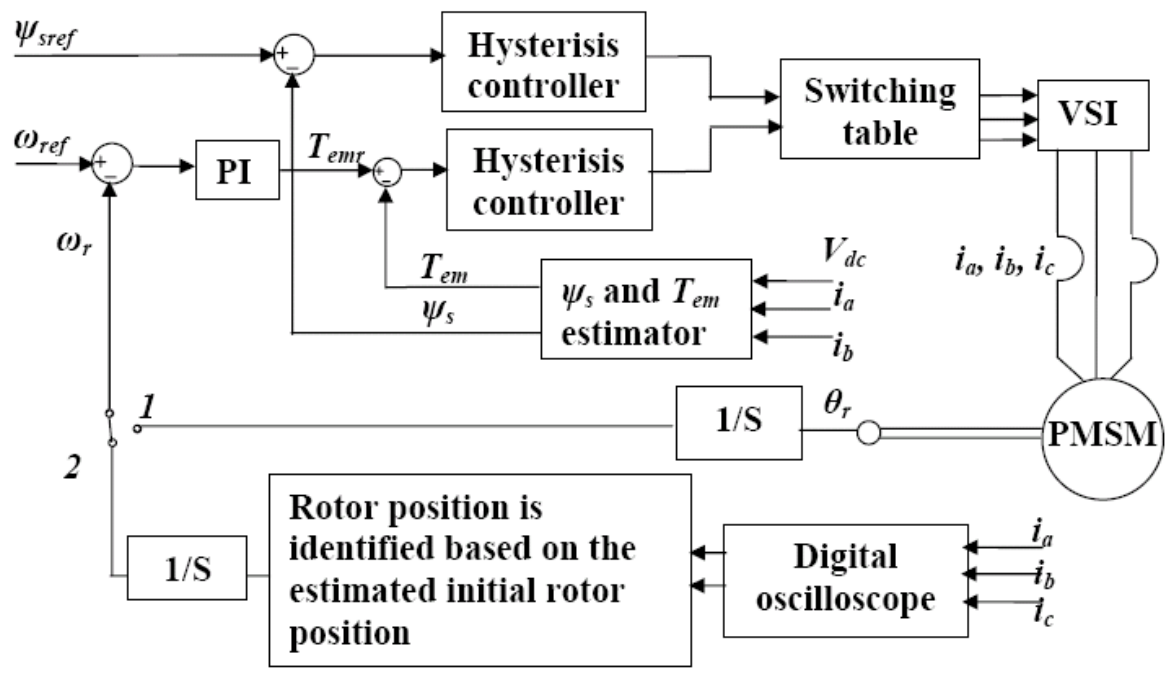

Fig. 9. Block diagram of the DTC system with the proposed rotor position estimation scheme.

\subsection{Experimental system configuration}

In order to verify the performance of the DTC drive, experiments should be carried out. The testing system consists of the testing surface mounted PMSM, a torque transducer, and a DC motor as the load coupled to the same shaft. The torque transducer is installed between the testing PMSM and the DC motor to monitor the load torque and rotor speed. The parameters of the PMSM have been listed in Table 1, and experiments are firstly conducted to measure several required electrical and magnetic parameters, such as the magnetising flux linkage (flux linkage produced by permanent magnets), stator winding resistance and inductances. The magnetising flux linkage $\psi_{p m}$ was obtained by measuring the phase voltage in the open circuit 
test as $\psi_{p m}=0.118 \mathrm{~Wb}$. The stator winding resistances at various temperatures were measured by the V-A method in at emperature controllable chamber, and the results are: $R_{a}=1.579 *(1+0.004033 T), R_{b}=1.584 *(1+0.004017 T)$, and $R_{c}=1.602 *(1+0.003946 T)$, where $T$ is the temperature in ${ }^{\circ} \mathrm{C}$. The inertia of the motor is $1.99 \times 10^{-3} \mathrm{~kg} \cdot \mathrm{m}^{2}$.

The PWM voltage source inverter (VSI) system is composed of six IGBTs connected to a DC power supply. The gate signals of the inverters are controlled by the dSPACE DS1104 control board, and the slave DSP subsystem is TMS320F240. Three phase current and DC bus voltage signals are detected by current and voltage sensors and are then sent to the dSAPCE DS1 104 system through analog-to-digital (A/D) converters.

\subsection{Experimental results}

Experiments have been conducted with the testing system for confirming the effectiveness of the presented rotor position identification strategy. Figs. 10 and $11 \mathrm{i}$ llustrate the experimental curves of the speed and torque during no-load start-up, respectively. Fig. 12 plots the stator flux trajectory at the steady state, and Fig. 13 shows the experimental stator currents.

At an estimated initial rotor position, e.g. $\theta=0^{\circ}$, the steady state performances of the experiment with a load torque of $3.0 \mathrm{Nm}$ and a reference speed of $2000 \mathrm{rpm}$ are obtained. Fig. 14 shows the rotor speed with an estimation scheme [14], electromagnetic torque, and stator flux trajectory, respectively. 


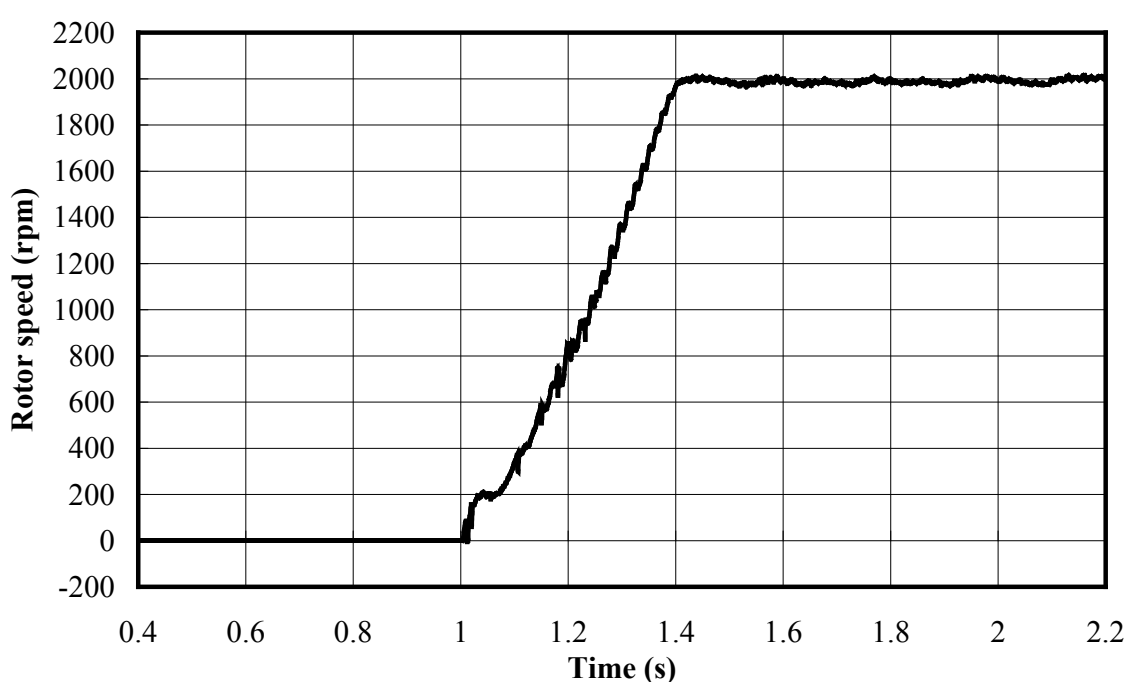

Fig. 10. Experimental speed curve of the sensorless DTC PMSM during no-load start-up

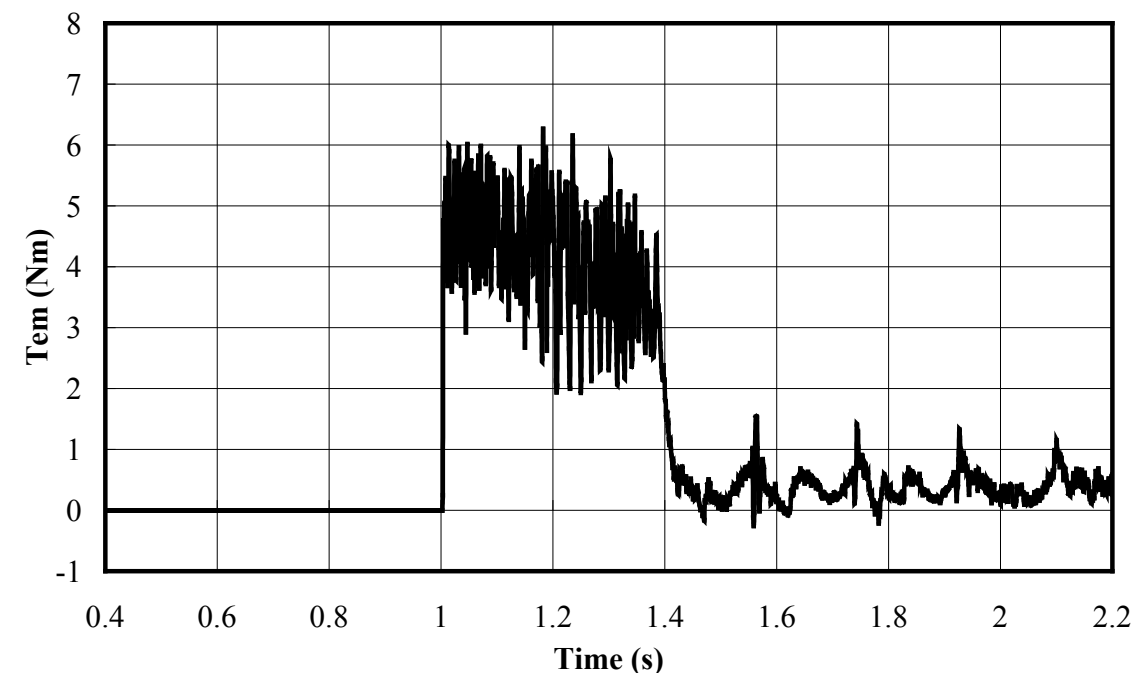

Fig. 11. Experimental electromagnetic torque of the sensorless DTC PMSM during no-load start-up 


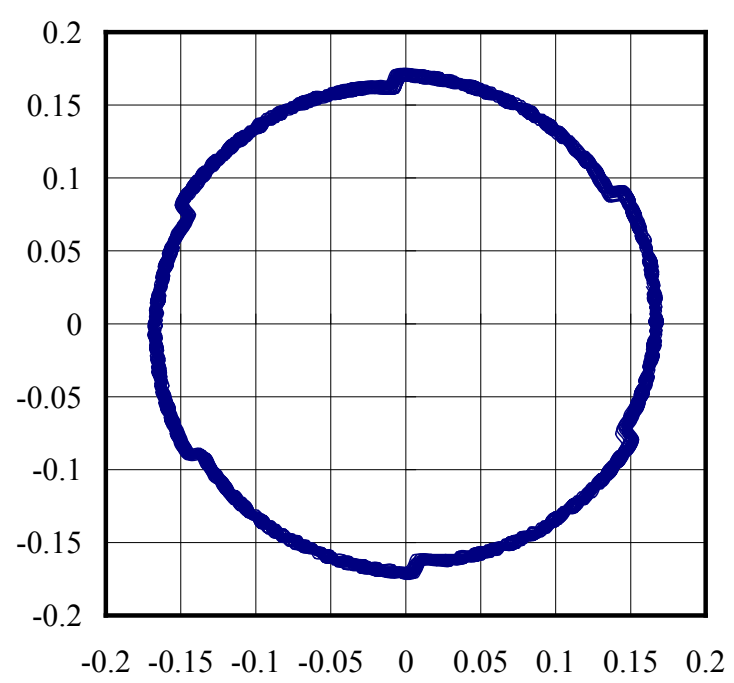

Fig. 12. Experimental stator flux trajectory of the sensorless DTC PMSM at steady state

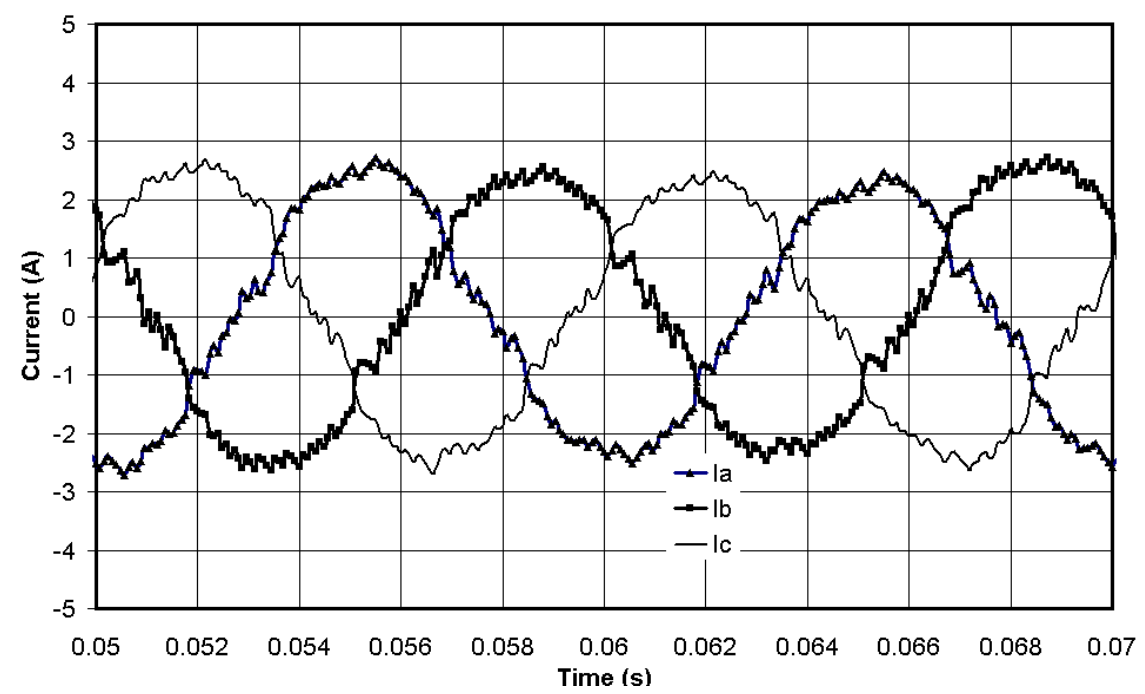

Fig. 13. Experimental stator currents of the sensorless DTC PMSM at steady state 


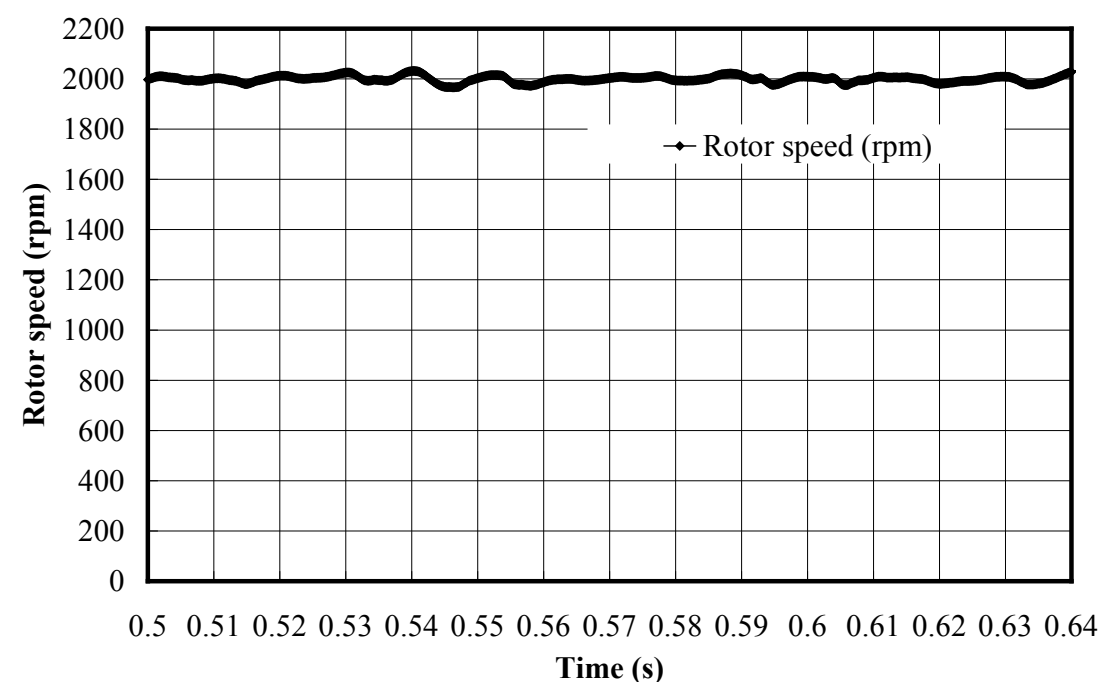

(a)

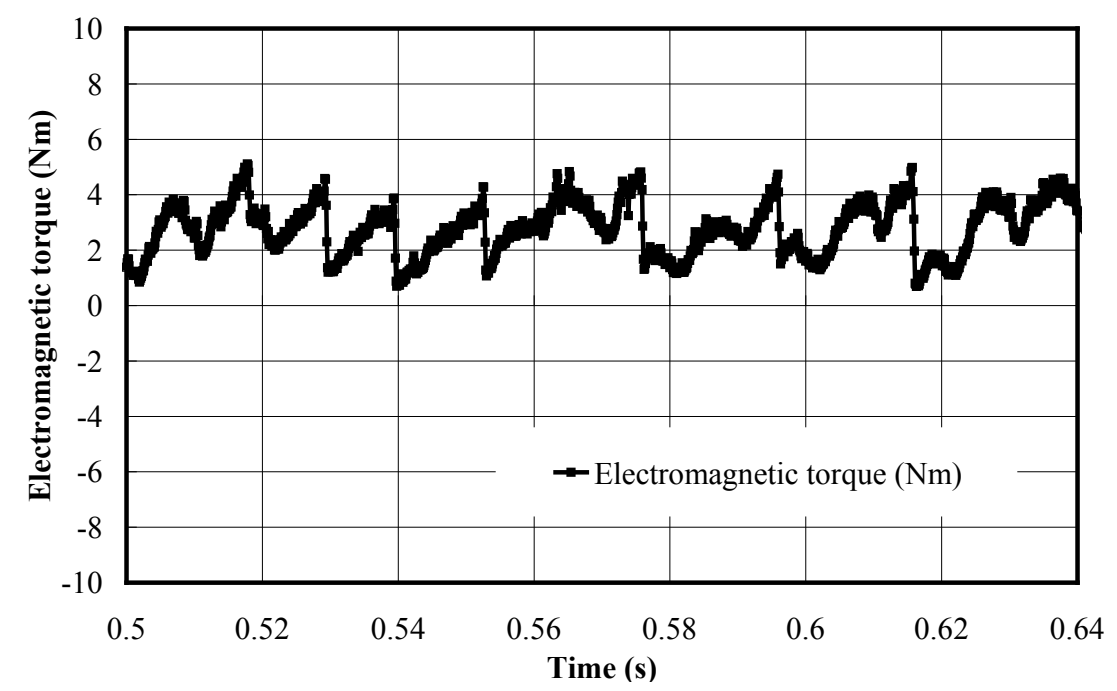

(b) 


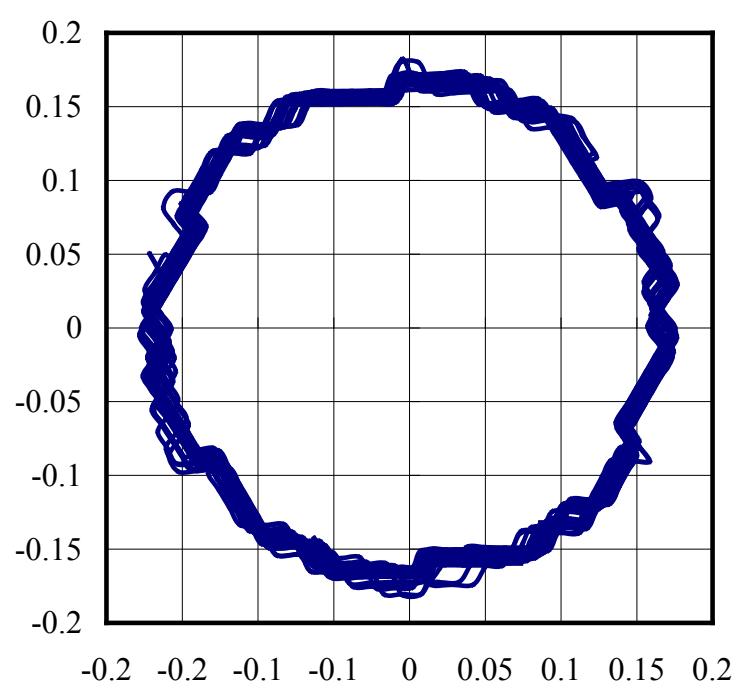

(c)

Fig. 14. Experimental steady state results of the sensorless PMSM DTC system with a load torque of 3.0 Nm:

(a) estimated rotor speed, (b) electromagnetic torque, and (c) stator flux trajectory.

\section{Conclusions}

In this paper, an initial rotor position estimation strategy for a DTC PMSM drive is presented. In the scheme, high voltage pulses are applied to amplify the saturation saliencies and corresponding peak currents are used, in combination with the inductance patterns, to determine the $d$-axis position and polarity of the rotor. The scheme can be simulated by using a nonlinear model of PMSM incorporating both structural and saturation saliencies, which are reflected in the winding inductances. The presented strategy has been implemented in a sensorless DTC scheme for a surface mounted PMSM. Experiments of the DTC system have been conducted. The effectiveness of the method and the performance of the DTC drive system have been confirmed by the experimental results. 


\section{Reference:}

[1] Acarnley, P. P., and Watson, J. F.: 'Review of position-sensorless operation of brushless permanent-magnet machines', IEEE Transactions on Industrial Electronics, 2006, 53, (2), pp. 352- 362

[2] Schroedl, M.: 'Sensorless control of ac machines at low speed and standstill based on the "INFORM" method', Proc. IEEE Industry Application Society Annual Meeting, San Diego, USA, Oct. 1996, pp. 270-277

[3] Corley M. J., and Lorenz, R. D.: 'Rotor position and velocity estimation for a permanent magnet synchronous machine at standstill and high speeds', Proc. IEEE Industry Application Society Annual Meeting, San Diego, USA, Oct. 1996, pp. 36-41

[4] Jang, J. H., Sul, S. K., Ha, J. I., Ide, K., and Sawamura, M.: 'Sensorless drive of surface-mounted permanent-magnet motor by high-frequency signal injection based on magnetic saliency', IEEE Transactions on Industry Applications, 2003, 39, (4), pp. 1031 -1039

[5] Nakashima, S., Inagaki, Y., and Miki, I.: 'Sensorless initial rotor position estimation of surface permanent-magnet synchronous motor', IEEE Transactions on I ndustry Applications, 2000, 36, (6), pp. 1598 - 1603

[6] Matsui, N., and Takeshita, T., 'A novel starting method of sensorless salient-pole brushless motor', Proc. IEEE Industry Application Society Annual Meeting, Denver, USA, Oct. 1994, pp. $386-392$

[7] Schmidt, P. B., Gasperi, M. L., Ray, G., and Wijenayake, A. H.: 'Initial rotor angle detection of a nonsalient pole permanent magnet synchronous machine', Proc. IEEE Industry Application Society Annual Meeting, New Orleans, USA, Oct. 1997, pp. 459-463

[8] Schmidt, P. B.: 'Method and apparatus for rotor angle detection'. U.S. Patent 6172 498, Jan. 2001 
[9] Yan Y., Zhu, J. G., Lu, H. W., Guo, Y. G., and Wang, S. H.: 'A PMSM model incorporating structural and saturation saliencies', Proc. International Conference on Electrical Machines and Systems, Nanjing, China, 27-29 Sept. 2005, pp. 194-199

[10] Yan, Zhu, J. G., Lu, H. W., Guo, Y. G., and Wang, S. H.: 'Study of a PMSM model incorporating structural and saturation saliencies', Proc. IEEE Int. Conf. on Power Electronics and Drive Systems, Kuala Lumpur, Malaysia, 28 Nov. - 1 Dec. 2005, pp. 575-580

[11] Yan Y., and Zhu, J.G.: 'Simulation of a direct torque controlled PMSM drive incorporating structural and saturation saliencies', Proc. IEEE Power Electronics Specialists Conf., Jiju Island, Korea, 18-22 June 2006, pp. 1-6

[12] Yan, Y., Zhu, J. G., Guo, Y. G., and Lu, H. W.: 'Modeling and simulation of direct torque controlled PMSM drive system incorporating structural and saturation saliencies', Proc. IEEE Industry Applications Society Annual Meeting, Tampa, USA, Oct. 2006, pp. 76-83

[13] Cui, P., Zhu, J. G., Ha, Q. P., Hunter, G. P., and Ramsden, V. S., 'Simulation of non-linear switched reluctance motor drive with PSIM', Proc. Int. Conf. on Electrical Machines and Systems, Shengyang, China, Aug. 2001, pp. 1061-1064

[14] Sun, D.: 'High performance direct torque control for permanent magnet synchronous motors'. Ph.D. Thesis, Zhejiang University, China, Mar. 2004

Ying Yan was born in Hebei, China. She received the B.E. degree in 2002 from North China Electric Power University. She is currently a Ph.D candidature in the Centre for Electrical Machines and Power Electronics, Faculty of Engineering, University of Technology, Sydney, Australia. Her research interests are: electrical machines and drives, and power system analysis. 
Jian Guo Zhu is the Professor of Electrical Engineering and Director for the Centre for Electrical Machines and Power Electronics at University of Technology, Sydney (UTS), Australia. He received his BE in 1982 from Jiangsu Institute of Technology, China, ME in 1987 from Shanghai University of Technology, China, and Ph.D in $1995 \mathrm{f}$ rom UTS, Australia. His research interests are: electromagnetics, magnetic properties of materials, electrical machines and drives, power electronics, and renewable energy systems.

YouGuang Guo received his B.E. degree from Huazhong University of Science and Technology (HUST), China in 1985, the M.E. degree from Zhejiang University, China in 1988, and the PhD degree from University of Technology, Sydney (UTS), Australia in 2004, all in electrical engineering.

From 1988 to 1998, he lectured in the Department of Electrical Engineering, HUST. From March 1998 to July 2000, he was a visiting research fellow in the Centre for Electrical Machines and Power Electronics, Faculty of Engineering, UTS. He is currently an ARC (Australia Research Council) postdoctoral research fellow at UTS.

His research fields include measurement and modelling of magnetic properties of magnetic materials, numerical analysis of electromagnetic field, motor design and optimisation, power electronics and control of electrical appliance. In these fields, he has published over 150 technical papers including 77 journal articles. 